\title{
Síndrome de Burnout em professores de Medicina: Uma revisão sistemática
}

\author{
Burnout Syndrome in Medicine teachers: A systematic review \\ Síndrome de Burnout en profesores de Medicina: Una revisión sistemática
}

Recebido: 28/04/2021 | Revisado: 05/05/2021 |Aceito: 09/05/2021 | Publicado: 18/05/2021

\author{
Miguel Vieira Gomes \\ ORCID: https://orcid.org/0000-0002-0851-2268 \\ Universidade Tiradentes, Brasil \\ E-mail: miguel.vieira@ souunit.com.br \\ Juliana Leal Freitas Maia \\ ORCID: https://orcid.org/0000-0002-1751-4572 \\ Universidade Tiradentes, Brasil \\ E-mail: juliana.maia@ souunit.com.br
}

\begin{abstract}
Resumo
Objetivo: Identificar a prevalência e fatores de risco para o desenvolvimento da síndrome de Burnout (SB) em professores do curso de medicina, bem como seus reflexos na qualidade de vida desses indivíduos. Métodos: Trata-se de uma revisão sistemática acerca da prevalência de SB em docentes do curso de medicina. O presente estudo baseouse nos princípios metodológicos para busca de artigos nas bases de dados eletrônicas: LILACS, PubMed, SciELO e plataforma Google Acadêmico, com corte temporal de 10 anos (2011-2021). Para o rastreamento dos artigos indexados nas bases de dados foram utilizados os descritores: "Burnout, Professional" e "Faculty, Medical". Resultados: Incluíram-se 15 artigos, em português, inglês e espanhol. Os artigos abordam a prevalência, consequências, fatores de risco e fatores protetores para a SB em docentes médicos. Conclusão: A SB, em geral, apresenta alta prevalência entre os docentes de medicina. Os estudos demonstram que os professores do curso de medicina estão em condição de relativa vulnerabilidade para o esgotamento mental, pois estão expostos a diversos fatores de risco para o desenvolvimento da SB. Dessa forma, estão relacionados ao aumento da exaustão emocional e despersonalização, resultando em menor realização profissional. Além disso, foi possível estabelecer uma correlação negativa entre Burnout e qualidade de vida dos docentes médicos. Todavia, é necessário maior padronização metodológica e mais estudos na área a fim de se estabelecer melhor o impacto dessa problemática no processo de ensino-aprendizagem e/ou definição de estratégias de prevenção do adoecimento mental dos docentes.
\end{abstract}

Palavras-chave: Burnout profissional; Docentes de medicina; Ensino em saúde.

\begin{abstract}
Objective: To identify the prevalence and risk factors for the development of Burnout syndrome (BS) in medical professors, as well as their reflexes on quality of life. Methods: This is a systematic review of the prevalence of BS in medical professors. The present study was based on the methodological principles for searching for articles in the electronic databases: LILACS, PubMed, SciELO and the Google Scholar platform, with a 10-year time cut (20112021). The following descriptors were used to track articles indexed in the databases: "Burnout, Professional" and "Faculty, Medical". Results: Were included 15 articles, in Portuguese, English and Spanish. The articles address the prevalence, consequences, risk factors and protective factors for BS in medical professors. Conclusion: BS, in general, has a high prevalence among medical professors. Studies show that medical school teachers are in a relatively vulnerable condition for mental exhaustion, as they are exposed to several risk factors for the development of BS. Thus, they are related to increased emotional exhaustion and depersonalization, resulting in less professional achievement. In addition, it was possible to establish a negative correlation between Burnout and the quality of life of medical professors. However, greater methodological standardization and more studies in the area are necessary in order to better establish the impact of this problem on the teaching-learning process and / or the definition of strategies for preventing teachers' mental illness.
\end{abstract}

Keywords: Burnout, Professional; Faculty, Medical; Health on Education.

\section{Resumen}

Objetivo: Identificar la prevalencia y los factores de riesgo para el desarrollo del síndrome de Burnout (SB) en profesores de facultades de medicina, así como sus reflejos sobre lacalidad de vida de estos individuos. Métodos: Se trata de una revisión sistemática de la prevalencia de SB en profesores de facultades de medicina. El presente estudio se basó em los principios metodológicos para la búsqueda de artículos em las bases de datos electrónicas: LILACS, PubMed, SciELO y la plataforma Google Scholar, con un corte temporal de 10 años (2011-2021). Para el seguimiento de los artículos indexados en las bases de datos se utilizaron los siguientes descriptores: "Burnout, Professional" y "Faculty, Medical". Resultados: Se incluyeron 15 artículos, en portugués, inglés y español. Los artículos abordan la 
prevalencia, las consecuencias, los factores de riesgo y los factores protectores de la SB en profesores de medicina. Conclusión: El SB, en general, tiene una alta prevalencia entre los profesores de medicina. Los estúdios muestran que los profesores de las facultades de medicina se encuentran en una condición relativamente vulnerable al agotamiento mental, ya que están expuestos a varios factores de riesgo para el desarrollo de BS. Por lo tanto, se relacionan conun mayor agotamiento emocional y despersonalización, lo que se traduce en un menor logro profesional. Además, se pudo establecer una correlación negativa entre Burnout y lacalidad de vida de los profesores de medicina. Sin embargo, es necesaria una mayor estandarización metodologico y más estudios em el área para estabelecer mejor el impacto de esta problemática em el proceso de enseñanza-aprendizaje y / o la definición de estrategias de prevención de la enfermedad mental docente.

Palabras clave: Agotamiento profesional; Docentes médicos; Educación para la salud.

\section{Introdução}

O trabalho é essencial para o homem. Seja por promover status, melhora da autoestima ou devido a necessidade de prover o sustento familiar (Silva et al., 2020). Por isso, as relações trabalhistas apresentam grandes implicações na vida do indivíduo, principalmente por demandar a maior parte do tempo e do convívio social, gerando exaustão e insatisfação (Dias et al., 2019).

Atualmente, os avanços nos modelos laborais exigem cada vez mais dedicação dos trabalhadores, além de impor maior competitividade e pressão por um aprimoramento constante, buscando os ideais de perfeição (Caixeta et al., 2021). O estresse ocupacional pode acarretar em conflitos, ansiedade, angústia e desestabilização emocional, contribuindo de forma negativa para o desenvolvimento de transtornos mentais bastante prevalentes na população (Junior et al., 2017).

Nesse contexto, destaca-se a síndrome de Burnout (SB), ou síndrome do esgotamento profissional, que surge como uma resposta crônica aos estressores emocionais e interpessoais no ambiente laboral (Tijdink et al., 2014). Essa patologia é compreendida em três tópicos pertinentes: a exaustão emocional, a despersonalização e a baixa realização profissional (Nazari et al., 2016).

A exaustão emocional é descrita como a resposta ao estresse individual, cursando com anergia e sensação de esgotamento total dos recursos emocionais e físicos (Pereira et al., 2019). A despersonalização se refere às relações interpessoais de forma negativa, apresentando distanciamento e falta de comprometimento com os resultados, tendo como consequências irritação, ansiedade e falta de motivação com as relações trabalhistas. Já a baixa realização profissional se caracteriza pela incapacidade do indivíduo de enxergar positivamente seu desempenho pessoal e profissional, levando a diminuição da autoestima, infelicidade e insatisfação (Lima et al., 2019).

Pesquisas indicam que o esgotamento profissional é prejudicial à saúde física e mental. Nesses indivíduos, podem ser observados sintomas físicos como tensão muscular, cefaleias e hipertensão, decorrentes do estresse causado pela tensão ocupacional e os desequilíbrios fisiológicos e psicológicos (Dos Santos et al., 2016). Além do mais, estão associados a irritabilidade, perda do entusiasmo e, em específico para os docentes, atitudes negativas com os estudantes (Yao et al., 2015).

As principais classes expostas ao estresse ocupacional crônico têm como característica comum lidar diretamente com pessoas, dessa forma apresentam maior risco para o desenvolvimento da SB (Caixeta et al., 2021). Um grande exemplo disso são os profissionais de saúde, pois se relacionam com as doenças da sociedade, com as demandas dos indivíduos que requerem auxílio e com as próprias demandas. Bem como os docentes, que também atuam nas relações interpessoais e são responsáveis pelo difícil e exaustivo trabalho de formação intelectual e desenvolvimento dos estudantes (Junior et al., 2017; Yao et al., 2015).

Alguns estudos sugerem que a SB e principalmente a exaustão emocional, é mais comum em médicos, influenciando na moral e produtividade, mas também reduzindo a qualidade de vida e elevando o risco de erros médicos (Tijdink et al., 2014). A prevalência da SB em profissionais da área de saúde varia entre $19 \%$ a 80,5\%, a depender da forma de aferição, país de estudo e especialidades. Sendo assim, os dados acerca da SB e seus preditores vem ganhando importância nas últimas 
décadas em consequência dos altos custos sociais e individuais, como depressão, ansiedade, dificuldades para dormir, baixo desempenho no trabalho, falta de empatia e agressividade (Popa-Velea et al., 2019).

$\mathrm{O}$ ambiente de trabalho expõe os professores a diversos fatores que influenciam para o esgotamento profissional, como atividades complexas, tensão emocional, atribuições específicas do processo de ensino-aprendizagem, cansaço físico e mental (Dos Santos et al., 2016). Entretanto, ao relacionar docência e o curso de medicina, pode-se observar um nível de desgaste ainda maior dos indivíduos, devido à alta responsabilidade, a autonomia profissional, o contato constante com os pacientes, a complexidades das relações e a exigência constante quanto às competências e habilidades (Dos Santos et al., 2016).

O convívio com adversidades estruturais, como a falta de materiais básicos, infraestrutura inadequada e alta demanda de atividades podem favorecer para o baixo desempenho, aumento da rotatividade, absenteísmo e dificuldade em se relacionar com os demais profissionais (Mendonça et al., 2012; Dos Santos et al., 2016). Ademais, existem estudos demonstrando que a falta de cuidado com a saúde dos funcionários pode causar sérios danos econômicos para as próprias instituições (Dias et al., 2019).

Nesse sentido, a pesquisa em questão justifica-se tendo em mente a alta prevalência da síndrome de Burnout e o esgotamento profissional relacionado aos docentes de medicina, bem como a alta exposição destes aos fatores de risco, os possíveis danos à saúde física e mental dos trabalhadores e os danos financeiros para as instituições (Junior et al., 2017; Dias et al., 2019). Além de que fica evidente a importância de medidas preventivas e curativas que visem melhorias nas condições de trabalho e maior qualidade de vida para os profissionais.

Com base nesse cenário, a pesquisa buscou identificar a prevalência e fatores de risco para o desenvolvimento da síndrome de Burnout em professores do curso de medicina, bem como seus reflexos na qualidade de vida desses indivíduos.

\section{Metodologia}

Trata-se de uma revisão sistemática acerca da prevalência de Síndrome de Burnout em docentes do curso de medicina. O levantamento sistematizado ocorreu entre os meses de março e abril de 2021. O presente estudo baseou-se nos princípios metodológicos para busca de artigos nas bases de dados eletrônicas: Literatura Latino-Americana e do Caribe em Ciências da Saúde (LILACS), US National Library of Medicine/National Institutes Of Health (PubMed), Scientific Electronic Library Online (Scielo) e plataforma Google Acadêmico.

Esta revisão sistemática é considerada de natureza qualitativa por se tratar de uma síntese de estudos pertinentes ao tema principal da mesma, com agrupamento e interpretação dos resultados de outros autores, porém sem aplicação de análises estatísticas de forma integral. Esse tipo de estudo tem como objetivo explorar as semelhanças e diferenças relevantes encontradas na literatura, visando potencializar a análise dos dados obtidos (Gomes \& Caminha, 2014; Pereira A. S. et al., 2018).

Para o rastreamento dos artigos indexados nas bases de dados mencionadas acima foram utilizados os descritores cadastrados em Ciências da Saúde (DeCS) da BVS (Biblioteca Virtual de Saúde) que compõem o tema: "Burnout, Professional" e "Faculty, Medical", sendo estes pesquisados de forma associada, utilizando o operador booleano AND.

Para eleger os artigos de interesse foi realizada uma leitura prévia do título e resumo, em que foram selecionados os textos com informações proveitosas para o trabalho. Em seguida, foram selecionadas as obras de interesse de acordo com os critérios de inclusão e exclusão definidos anteriormente a partir da leitura seletiva dos resumos. E, por fim, a etapa de extração de dados, que permitiu analisar minuciosamente os artigos na íntegra, selecionando aquelas publicações que tratavam de assuntos relevantes ao tema.

Os critérios de inclusão definidos para seleção dos artigos foram: artigos publicados em português, inglês e espanhol; 
que abordassem a Síndrome de Burnout em docentes de medicina; artigos completos e disponíveis, sendo citados no mínimo dois descritores utilizados no estudo e aqueles publicados nos últimos dez anos (2011-2021).

$\mathrm{Na}$ base de dados PubMed, combinaram-se os descritores da seguinte forma: (Burnout, Professional) AND (Faculty, Medical), obtendo-se 553 artigos. Foram selecionados 9 artigos a partir da leitura de títulos e resumos. Sendo feita a leitura na íntegra e considerando critérios de inclusão e exclusão, 5 artigos foram selecionados para estudo.

Já na base de dados LILACS, a combinação dos descritores citados acima resultou em 13 artigos. Após a leitura dos títulos e resumos, foram selecionados 3 estudos para a leitura na íntegra. Entretanto, depois da análise na íntegra e aplicação dos critérios de inclusão e exclusão, 2 artigos foram selecionados para estudo.

Na plataforma SciELO, foram encontrados 2 artigos de acordo com a busca a partir dos descritores supracitados. Porém, ao realizar a leitura dos títulos e resumos, nenhum artigo desta base de dados atendeu aos critérios de inclusão e exclusão do estudo.

Destaca-se também que, devido ao baixo número de artigos encontrados nas plataformas citadas previamente, realizou-se busca manual no Google Acadêmico utilizando os descritores: Burnout, Professional AND Faculty, Medical. Dessa forma, foram analisados os estudos encontrados e selecionados um total de 8 , sendo esses os que se adequaram aos critérios de inclusão e exclusão propostos pela pesquisa.

Com isso, foram selecionados no total 20 artigos para leitura de texto completo, sendo 15 deles incluídos para integrar esta revisão sistemática. A seguir apresenta-se a Figura 1, que mostra o fluxograma de descrição das etapas de seleção dos estudos.

Figura 1 - Fluxograma com as etapas de seleção dos artigos.

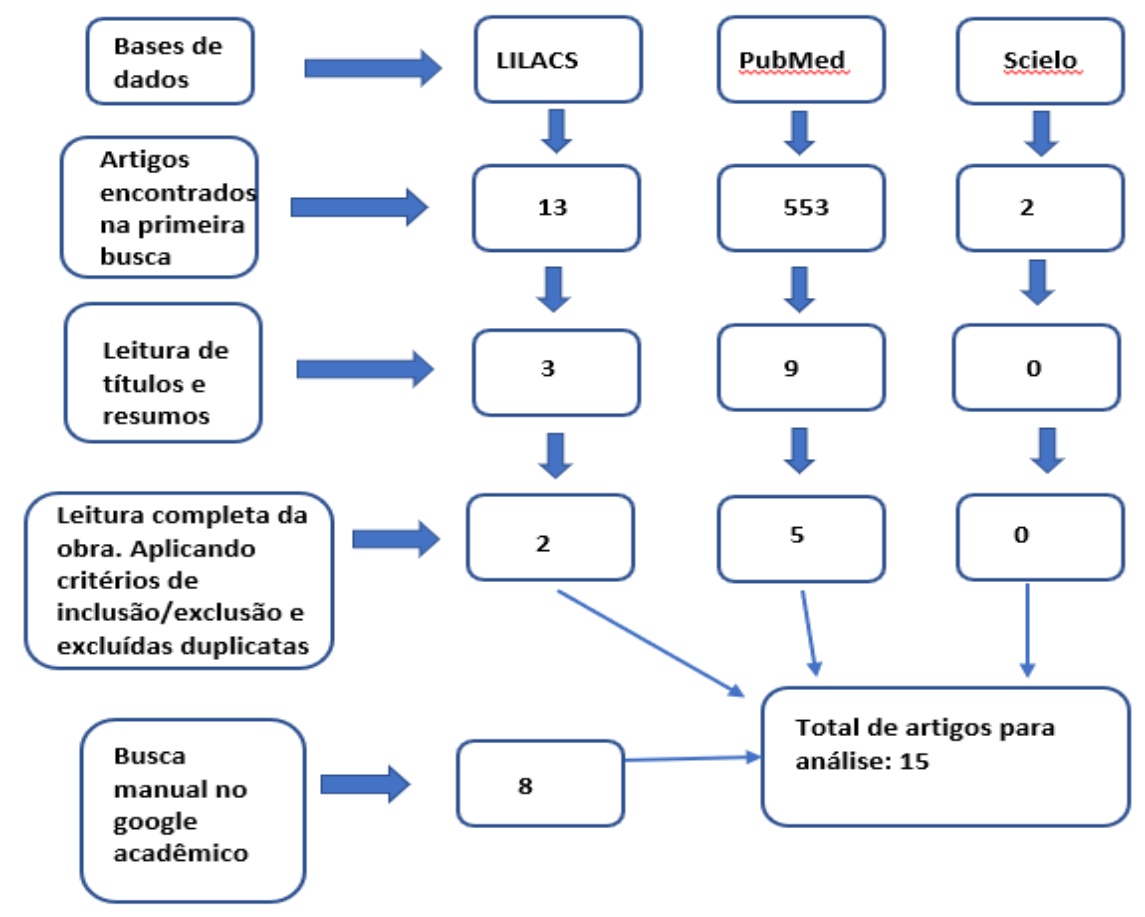

Fonte: Autores (2021).

Foram excluídos dos trabalhos os artigos não indexados nas bases de dados; teses; capítulos de livros; livros; relatório técnico; artigos duplicados, bem como aqueles que disponibilizaram somente os resumos da publicação ou estudos que não 
atendiam o objetivo da pesquisa.

\section{Resultados}

Após seleção dos estudos, eles foram organizados de acordo com os autores, ano de publicação, objetivos e resultados. Os artigos foram publicados em português, inglês e espanhol. Com relação ao ano de publicação, 2011 apresenta dois (13,33\%) artigos publicados; 2012 com um (6,66\%) artigos; 2014 com dois artigos (13,33\%); 2014, dois artigos (13,33\%); 2017, um artigo (6,66\%); 2019, quatro artigos (26,66\%); 2020, dois artigos (13,33\%); e 2021, um artigo (6,66\%). A Tabela 1 mostra os 15 artigos selecionados e organizados conforme explicitado anteriormente.

Tabela 1 - Artigos recuperados na revisão sistemática.

\begin{tabular}{|c|c|c|c|}
\hline Autor & $\begin{array}{c}\text { Ano de } \\
\text { publicação }\end{array}$ & Objetivos & Resultados \\
\hline $\begin{array}{l}\text { Caixeta } \text { et al., } \\
\qquad(2021)\end{array}$ & 2021 & $\begin{array}{c}\text { Verificar a incidência da SB } \\
\text { entre as profissões na } \\
\text { contemporaneidade e relacionar com as } \\
\text { causas e consequências decorrentes } \\
\text { dela }\end{array}$ & $\begin{array}{l}\text { Os artigos revisados revelam evidências expressivas da SB } \\
\text { nas seguintes profissões: profissionais da saúde } \\
\text { (enfermeiros, médicos, fisioterapeutas, motoristas do } \\
\text { transporte de saúde, educadores físicos, gerentes da ESF); } \\
\text { policiais militares, bombeiros, professores, bancários e } \\
\text { motoristas de transporte coletivo. }\end{array}$ \\
\hline Duke et al., (2020) & 2020 & $\begin{array}{l}\text { Analisar a relação entre o contexto } \\
\text { institucional e as características da } \\
\text { síndrome de Burnout, como um meio } \\
\text { de identificar oportunidades para que as } \\
\text { lideranças organizacionais abordem o } \\
\text { Burnout ligado a faculdade }\end{array}$ & $\begin{array}{c}\text { Embora muito do foco em abordar o esgotamento em } \\
\text { ambientes de saúde tenha sido a promoção de habilidades de } \\
\text { enfrentamento e construção de resiliência no nível } \\
\text { individual, nossas descobertas adicionam a uma literatura } \\
\text { crescente que documenta um papel significativo para a } \\
\text { liderança institucional na identificação e facilitação de } \\
\text { estratégias para promover o bem-estar do corpo docente. } \\
\text { As descobertas também apoiam o papel da liderança para } \\
\text { melhorar o clima institucional por meio da criação de } \\
\text { oportunidades para aumentar as percepções do corpo } \\
\text { docente de capacitação e valor no departamento. }\end{array}$ \\
\hline Silva et al., (2020) & 2020 & $\begin{array}{l}\text { Identificar os indicadores de Síndrome } \\
\text { de Burnout entre docentes de um centro } \\
\text { universitário do Acre. }\end{array}$ & $\begin{array}{l}\text { Os resultados apontam que existe a possibilidade de que a } \\
\text { síndrome esteja em processo de inicialização na população } \\
\text { estudada, tendo em vista que os resultados obtidos nos } \\
\text { quesitos de exaustão emocional e despersonalização foram } \\
\text { relevantes, no entanto o desenvolvimento da doença pode } \\
\text { estar sendo contido pelo sentimento de realização } \\
\text { profissional dos indivíduos. }\end{array}$ \\
\hline Dias et al., (2019) & 2019 & $\begin{array}{l}\text { Avaliar a prevalência da SB nos } \\
\text { docentes do curso de Medicina de uma } \\
\text { faculdade privada, relacionando-a com } \\
\text { outras variáveis, incluindo a } \\
\text { religiosidade. }\end{array}$ & $\begin{array}{l}\text { Considerando um dos critérios utilizados, } 5 \text { docentes } \\
(3,88 \%) \text { foram diagnosticados com } \mathrm{SB} \text {, sendo todos do sexo } \\
\text { masculino. Além disso, os escores da escala de DUREL } \\
\text { relativos aos itens RI1e RI2 foram maiores entre os } \\
\text { portadores da síndrome, indicando menor nível de }\end{array}$ \\
\hline
\end{tabular}


Research, Society and Development, v. 10, n. 6, e12710615578, 2021

(CC BY 4.0) | ISSN 2525-3409 | DOI: http://dx.doi.org/10.33448/rsd-v10i6.15578

\begin{tabular}{|c|c|c|c|}
\hline & & & $\begin{array}{l}\text { religiosidade. } \\
\text { Entretanto, utilizando outro critério, } 45(34,88 \%) \text { docentes } \\
\text { apresentam SB, sendo 73,33\% deles do sexo masculino, não } \\
\text { havendo diferença significativa entre os níveis de } \\
\text { religiosidade dos grupos com e sem Burnout. }\end{array}$ \\
\hline Lima et al., (2019) & 2019 & $\begin{array}{l}\text { Verificar a prevalência de Síndrome de } \\
\text { Burnout em professores } \\
\text { médicos de Instituição de Ensino } \\
\text { Superior do sertão da Paraíba. }\end{array}$ & $\begin{array}{l}\text { Foi descartada a prevalência de Síndrome de Burnout na } \\
\qquad \text { população pesquisada. } \\
\text { Não foi encontrada simultaneamente a presença de } \\
\text { esgotamento emocional, despersonalização e de realização } \\
\text { profissional. } \\
\text { As principais dimensões afetadas foram a de esgotamento } \\
\text { emocional e de despersonalização. } \\
\text { Não foram encontrados valores significativos de baixa } \\
\text { realização profissional }\end{array}$ \\
\hline $\begin{array}{l}\text { Pereira et al, } \\
\text { (2019) }\end{array}$ & 2019 & $\begin{array}{l}\text { Sistematizar o conhecimento disponível } \\
\text { na literatura acerca da Síndrome de } \\
\text { Burnout em docentes atuantes no curso } \\
\text { de medicina }\end{array}$ & $\begin{array}{l}\text { A maioria dos professores de medicina sofrem de Síndrome } \\
\text { de Burnout, em que os principais fatores que desencadeiam } \\
\text { tal síndrome são: estar em um estágio de início de carreira, o } \\
\text { fato de ter filhos em casa, jornada dupla de trabalho, } \\
\text { falta de valorização, baixa remuneração e estresse no } \\
\text { ambiente de trabalho. }\end{array}$ \\
\hline $\begin{array}{l}\text { Popa-Velea } \text { et al., } \\
\text { (2019) }\end{array}$ & 2019 & $\begin{array}{l}\text { Este estudo teve como objetivo avaliar } \\
\text { a extensão do Burnout em médicos } \\
\text { acadêmicos romenos e moldavos e } \\
\text { determinar o valor preditivo da } \\
\text { inteligência emocional, estratégias de } \\
\text { enfrentamento, motivação no trabalho, } \\
\text { suporte organizacional percebido e as } \\
\text { características sociodemográficas da } \\
\text { Burnout }\end{array}$ & $\begin{array}{l}\text { Os participantes da Moldávia tiveram pontuações } \\
\text { significativamente mais baixas em Burnout e amotivação e } \\
\text { pontuações mais altas em inteligência emocional, suporte } \\
\text { organizacional percebido e motivação no trabalho. } \\
\text { Os principais preditores de Burnout foram baixa motivação e } \\
\text { baixo suporte organizacional percebido nos entrevistados da } \\
\text { Moldávia e baixa motivação no trabalho nos participantes } \\
\text { Romenos. }\end{array}$ \\
\hline Junior et al., (2017) & 2017 & $\begin{array}{l}\text { O objetivo deste estudo é avaliar a } \\
\text { relação entre a síndrome de Burnout e a } \\
\text { prática docente no ensino médico na } \\
\text { instituição Faculdade São Lucas, Porto } \\
\text { Velho - Rondônia, Brasil. }\end{array}$ & $\begin{array}{l}\text { Nos resultados obtidos, observamos que } 13 \text { professores } \\
\text { (52\%) estão passíveis de desenvolver a síndrome, } 11 \text { (44\%) } \\
\text { se encontram na fase inicial da síndrome e } 1 \text { (4\%) já está na } \\
\text { fase considerável da Síndrome de Burnout. }\end{array}$ \\
\hline $\begin{array}{c}\text { Dos Santos et al., } \\
\text { (2016) }\end{array}$ & 2016 & $\begin{array}{l}\text { Conhecer a percepção dos docentes de } \\
\text { Enfermagem e Medicina da } \\
\text { Universidade Federal do Rio Grande do } \\
\text { Norte quanto aos estressores do } \\
\text { ambiente ocupacional. }\end{array}$ & $\begin{array}{l}\text { Com base na coleta de dados, foi possível caracterizar os } \\
\qquad \text { participantes da seguinte } \\
\text { forma: a maioria dos docentes é do sexo masculino, casados, } \\
\text { acima de } 40 \text { anos, com mais de } 10 \text { anos de formação, com } \\
\text { doutorado, em regime de dedicação exclusiva, e ministram } \\
\text { aulas, tanto na graduação quanto na pós-graduação. }\end{array}$ \\
\hline
\end{tabular}


Research, Society and Development, v. 10, n. 6, e12710615578, 2021

(CC BY 4.0) | ISSN 2525-3409 | DOI: http://dx.doi.org/10.33448/rsd-v10i6.15578

\begin{tabular}{|c|c|c|c|}
\hline $\begin{array}{l}\text { Nazari et al., } \\
\qquad(2016)\end{array}$ & 2016 & $\begin{array}{l}\text { O objetivo deste estudo foi determinar } \\
\text { o estresse ocupacional e sua relação } \\
\text { com a síndrome de Burnout em } \\
\text { membros da Lorestan University of } \\
\text { Medical Sciences. }\end{array}$ & $\begin{array}{l}\text { Os resultados mostraram que a maioria dos participantes } \\
\text { apresentou baixo nível de Burnout em três dimensões } \\
\text { incluindo Burnout emocional (72,1\%), despersonalização } \\
(81,1 \%) \text { e diminuição da realização pessoal }(56,8 \%) \text {. } \\
\text { Além disso, } 79,3 \% \text { das amostras apresentaram baixo estresse } \\
\text { ocupacional, mas houve uma relação significativa entre o } \\
\text { estresse ocupacional e as dimensões da síndrome de } \\
\text { Burnout, com exceção da intensidade de decréscimo da } \\
\text { realização pessoal. }\end{array}$ \\
\hline Yao et al., (2015) & 2015 & $\begin{array}{l}\text { Avaliar os níveis de Burnout e os } \\
\text { fatores contribuintes entre a equipe de } \\
\text { docentes de medicina na China } \\
\text { continental; e esclarecer a inter-relação } \\
\text { entre Burnout relacionado ao trabalho e } \\
\text { qualidade de vida. }\end{array}$ & $\begin{array}{l}\text { Os preditores mais significativos e comuns da prevenção do } \\
\text { Burnout foram o amor pela profissão docente e o } \\
\text { reconhecimento do trabalho por um supervisor direto. } \\
\text { O esgotamento relacionado ao trabalho teve um efeito } \\
\text { negativo direto na qualidade de vida. }\end{array}$ \\
\hline $\begin{array}{l}\text { Tijdink et al., } \\
\qquad(2014)\end{array}$ & 2014 & $\begin{array}{c}\text { Embora o Burnout relacionado ao } \\
\text { trabalho e sua característica central a } \\
\text { exaustão emocional sejam comuns } \\
\text { entre os profissionais médicos e } \\
\text { comprometam a satisfação no trabalho } \\
\text { e o desempenho profissional, eles } \\
\text { nunca foram sistematicamente } \\
\text { estudados em professores de medicina, } \\
\text { que ocupam posições centrais na } \\
\text { medicina acadêmica. }\end{array}$ & $\begin{array}{l}\text { Aproximadamente um quarto }(23,8 \%) \text { pontuou acima do } \\
\text { ponto de corte usado para a definição de exaustão } \\
\text { emocional. Fatores relacionados a estar em um estágio } \\
\text { inicial de carreira (ou seja, menor idade, menos anos desde a } \\
\text { nomeação, ter filhos que vivem em casa, ter um índice de } \\
\text { Hirsch relativamente baixo) foram significativamente } \\
\text { associados a maiores escores de exaustão emocional. } \\
\text { Houve uma correlação inversa significativa entre exaustão e } \\
\text { o nível de engajamento profissional. }\end{array}$ \\
\hline $\begin{array}{l}\text { Mendonça et al., } \\
\text { (2012) }\end{array}$ & 2012 & $\begin{array}{l}\text { Trata-se de um estudo correlacional e } \\
\text { de comparação entre participantes, em } \\
\text { que foram considerados dois conjuntos } \\
\text { principais de variáveis: critério } \\
\text { (síndrome de Burnout e fadiga) e } \\
\text { antecedentes (estresse no trabalho e } \\
\text { indicadores de situação de trabalho) }\end{array}$ & $\begin{array}{c}\text { Evidenciou-se que o estresse no trabalho tem implicações } \\
\text { negativas, como a síndrome de Burnout e a fadiga }\end{array}$ \\
\hline $\begin{array}{l}\text { Gonçalves et al., } \\
\qquad \text { (2011) }\end{array}$ & 2011 & $\begin{array}{l}\text { Analisar a prevalência da SB nos } \\
\text { professores médicos do } 1^{\circ} \text { ao } 4^{\circ} \text { ano do } \\
\text { curso de Medicina da Universidade do } \\
\text { Estado do Pará (UEPA), durante o ano } \\
\text { de } 2011\end{array}$ & $\begin{array}{l}\text { A média total de idade dos participantes foi } 50,55 \text { anos e } \\
\text { tempo médio de profissão de } 25,96 \text { anos. } \\
\text { Metade dos professores médicos apresentou a SB, dentre os } \\
\text { quais } 58,3 \% \text { pertenciam ao sexo feminino. } \\
\text { A dimensão que demonstrou ter os níveis mais altos entre os } \\
\text { pesquisados foi a despersonalização. }\end{array}$ \\
\hline
\end{tabular}




\begin{tabular}{|c|c|c|c|}
\hline $\begin{array}{l}\text { Portilho et al., } \\
\text { (2011) }\end{array}$ & 2011 & $\begin{array}{l}\text { Analisar as possíveis manifestações da } \\
\text { Síndrome de Burnout em vinte e dois } \\
\text { professores titulares e ocasionais dos } \\
\text { programas de medicina e serviço social } \\
\text { de uma universidade da região cafeeira } \\
\text { - Colômbia no primeiro semestre de } \\
2011\end{array}$ & $\begin{array}{l}\text { Verificou-se que o nível baixo foi de } 5 \% \text {, no nível médio foi } \\
\text { de } 82 \% \text { e no nível alto } 14 \% \text {, por sua vez, verificou-se que } \\
\text { em idade mais jovem há maior prevalência de apresentar } \\
\text { esta síndrome. }\end{array}$ \\
\hline
\end{tabular}

Fonte: Autores (2021).

\section{Discussão}

A síndrome de Burnout (SB) é um problema de saúde pública decorrente da relação entre o indivíduo e o ambiente de trabalho (Caixeta et al., 2021). Segundo Dos Santos et al., (2016), a SB é descrita por alguns autores como um processo de desgaste que se desenvolve ao longo do tempo; entretanto, outros escritores demonstram maior incidência nos profissionais que estão iniciando a carreira acadêmica. A falta de experiência na área, a ausência de formas de enfrentamento às situações e a pouca idade são identificadas como possíveis fatores predisponentes para o esgotamento profissional nesses casos. (Silva et al., 2020)

Conforme citado por Caixeta et al., (2021), em estudo que relaciona o Burnout com as profissões e suas consequências, foi observado que que os professores e os profissionais de saúde estão entre os mais acometidos. Longas jornadas de trabalho, desempenho de múltiplas funções e relações interpessoais estão entre os principais fatores que levam à exaustão. Entre os médicos, foi estimada uma prevalência entre 25 a $67 \%$, com impacto variável a depender da especialidade e atribuições. As incidências mais altas foram verificadas entre os profissionais que atuam em UTIs, devido a exposição contínua ao estresse e sobrecarga física e emocional. Já os dermatologistas e anestesistas, apresentaram menores índices de esgotamento. (Caixeta et al., 2021)

De acordo com Pereira et al., (2019), a maioria dos professores de medicina sofrem com o esgotamento profissional e apresentam maiores escores de exaustão emocional. Dentre outros fatores, identificou-se que atuar como professor e médico em jornada dupla, a falta de valorização proporcional ao grau de esforço, altas demandas e o estresse no ambiente de trabalho estão relacionadas ao aumento da exaustão emocional e despersonalização, resultando em menor realização profissional. Além disso, Mendonça et al., (2012) evidenciaram que sobrecarga de trabalho pode estar relacionada às atividades de ensino, produção científica e execução de funções administrativas.

Já Dos Santos et al., (2016), descreveram que a maioria dos participantes referem como contribuição para a falta de motivação: as cargas horárias elevadas e instáveis, os processos burocráticos, a desvalorização do papel do docente, a falta de interesse dos alunos e a falta de estrutura adequada. Consequentemente, os profissionais podem apresentar exaustão e o aparecimento de sintomas físicos, como taquicardia, sobrepeso e hipertensão, além do desenvolvimento de sintomas psicológicos e outros transtornos psiquiátricos.

Tijdink et al., (2014), identificaram que 104 dentre 437 docentes médicos na Holanda apresentaram escores compatíveis com o diagnóstico de Burnout, totalizando 23,8\% de prevalência da população estudada. De forma semelhante aos resultados encontrados por Portilho et al., (2011), nesse estudo foram descritos como fatores de risco indivíduos em início de carreira, com menor idade e, em alguns casos, com filhos.

A pesquisa desenvolvida por Gonçalves et al., (2011), realizada com 55 docentes médicos no Pará, observou elevada prevalência da SB (50\%). Além disso, foi visto que a média geral de idade foi de 50,79 anos e a carga horária média foi de 
48,83 horas semanais entre os participantes. Porém, ao analisar apenas os dados referentes aos indivíduos com Burnout encontrou-se médias de idade de 25,58 anos e carga horária média de 53 horas semanais. Dessa forma, apresenta concordância com os dados encontrados na literatura atual, indicando a maior prevalência de SB em docentes médicos e classificando como fatores de risco menor idade e maior carga horária de trabalho. (Tijdink et al., 2014; Pereira et al., 2019; Lima et al., 2019; Dos Santos et al., 2016)

Em contrapartida, o estudo realizado por Junior et al., (2017), com um total de 25 participantes, demonstrou que os professores de uma faculdade de medicina em Rondônia, de forma geral, apresentaram plenas condições para as demandas laborais e ausência de sobrecarga emocional. Porém, identificou que existem também participantes com risco médio e alto para o desenvolvimento da síndrome de Burnout, sugerindo que os professores médicos estão em uma condição de relativa vulnerabilidade para o esgotamento profissional. De forma semelhante, Lima et al., (2019) apresentou índice alto de esgotamento emocional (11,7\%), porém não demonstrou prevalência de SB entre os participantes. Segundo o autor, esse fato decorre da ausência de consenso sobre a metodologia que deve ser aplicada nas investigações relacionadas à síndrome, o que pode acarretar em valores distintos a depender da população estudada e o método de análise das informações.

Nazari et al., (2016), ao investigar 111 membros da Universidade de Ciências Médicas de Lorestão, no Irã, evidenciou correlação positiva entre estresse laboral e a síndrome de Burnout, porém, a maioria dos participantes apresentavam bons resultados nos tópicos referentes à exaustão emocional e despersonalização; já no tópico referente a realização pessoal demonstrou situação moderada a severa. Além disso, foi possível observar menor frequência e intensidade de esgotamento profissional nos professores dos ciclos básicos do que nos clínicos. Ademais, esse artigo, assim como o conduzido por Junior et al., (2017), inferiu maior vulnerabilidade para o desenvolvimento do esgotamento profissional de acordo com o nível de graduação, em especial profissionais com mestrado e doutorado.

Com relação aos gêneros, não há unanimidade na literatura atual para a definição de maior ou menor incidência de Burnout entre os sexos. Porém, genericamente, as mulheres apresentam maiores escores em exaustão emocional e os homens em despersonalização. Sendo assim, essa discordância pode estar relacionada aos diferentes papéis sociais e culturais dos sexos masculino e feminino. As mulheres tendem a se expressar mais livremente, com exposição de dificuldades e conflitos, porém com maior alívio de angústias, raivas ou indignações e, além disso, apresentam jornada dupla, no âmbito familiar e profissional. Já os homens, tendem a expressar seus sentimentos apenas após alcançar seus limites máximos ou em situações insuportáveis. (Junior et al., 2017; Yao et al., 2015)

O estudo conduzido por Yao et al., (2015), não evidenciou diferenças significativas nos domínios relacionados ao sexo, idade, nível educacional, estado civil, condições de vida, anos de ensino, horas semanais de ensino, horas de trabalho diárias, horas de sono por dia e estado de saúde. Por outro lado, os dados encontrados estabeleceram uma correlação negativa entre Burnout e qualidade de vida dos professores universitários. Esse fato pode ser influenciado pela baixa satisfação pela especialidade, ausência de atividades prazerosas, sobrecarga de trabalho, baixos salários e pouco reconhecimento por superiores. Foram considerados como fatores protetores o amor por ensinar e o reconhecimento por um supervisor direto, pois, nesses casos, os indivíduos apresentaram menores escores em todas as dimensões.

Os professores médicos, nas pesquisas realizadas por Lima et al., (2019) e por Dos Santos et al., (2016), consideraram as aulas teóricas e o reconhecimento dos alunos como algo prazeroso e que eleva a satisfação com a profissão. Além disso, veem a formação de uma rede de apoio, como ter filhos, cônjuge e a convivência em grupos fora do trabalho como fatores de proteção para o desenvolvimento do esgotamento profissional. De forma complementar, Duke et al., (2020) e Popa-Velea et al., (2019) descrevem que um ambiente de trabalho agradável, estruturado adequadamente, com maior flexibilidade e autonomia para os docentes contribuem para evitar o desgaste físico e emocional, promovendo maior saúde e bem-estar.

A religiosidade, apesar de pouco citada nas pesquisas, foi descrita por Dias et al., (2019) como um fator protetor ao 
desenvolvimento da SB. O autor aponta que os docentes médicos com o diagnóstico da síndrome, em geral, apresentaram menores índices de religiosidade intrínseca. Por outro lado, os religiosos veem a crença como algo superior às dificuldades individuais.

\section{Conclusão}

Os estudos demonstram que os professores do curso de medicina estão em condição de relativa vulnerabilidade para o esgotamento mental. Esses profissionais estão expostos a diversos fatores de risco para o desenvolvimento da SB, incluindo menor idade, início de carreira, cargas horárias elevadas e instáveis, relações interpessoais, desempenho de múltiplas funções, falta de valorização proporcional ao grau de esforço, estresse no ambiente de trabalho, processos burocráticos, falta de interesse dos alunos e a falta de estrutura adequada. Dessa forma, estão relacionados ao aumento da exaustão emocional e despersonalização, resultando em menor realização profissional.

Além disso, os dados encontrados estabeleceram uma correlação negativa entre Burnout e qualidade de vida dos docentes médicos. Consequentemente, esses profissionais podem apresentar exaustão e o aparecimento de sintomas físicos, como taquicardia, sobrepeso e hipertensão, além do desenvolvimento de sintomas psicológicos e outros transtornos psiquiátricos.

Por outro lado, foram considerados fatores protetores o amor pelo ensino, ter uma religião, o grau satisfação profissional, o nível de reconhecimento pelos alunos e superiores, formação de uma rede de apoio e convivência em grupos fora do trabalho.

Conclui-se, portanto, que a síndrome de Burnout, em geral, apresenta alta prevalência entre os docentes de medicina. Todavia, é possível inferir que não há consenso sobre as metodologias empregadas e, por isso, pode apresentar diferenças de acordo com a população e os métodos de análise utilizados. Além disso, é possível constatar que, a despeito do interesse crescente sobre o tema, ainda há escassez de informações científicas acerca da SB nos professores do curso de medicina. Por conseguinte, são necessários mais estudos na área, a fim de se estabelecer melhor o impacto dessa problemática no processo de ensino-aprendizagem, além de contribuir para a definição de estratégias de prevenção do adoecimento mental dos docentes.

É de grande relevância a identificação e o desenvolvimento de políticas que visem o bom relacionamento e bem-estar dos professores médicos, conciliando de forma benéfica a dupla jornada de trabalho, apresentando maior valorização do trabalhador, fornecendo maior assistência aos profissionais em início de carreira e potencializando os fatores protetores para cada indivíduo.

\section{Referências}

Caixeta, N. C. et al (2021). A síndrome de Burnout entre as profissões e suas consequências/Burnout. Brazilian Journal of Health Review, v. 4, n. 1, p. 593$610,2021$.

Dias, K. M. et al (2019). Prevalência da síndrome de Burnout em professores do curso de medicina de uma faculdade particular de Belo Horizonte. Revista Interdisciplinar de Ciências Médicas, v. 3, n. 2, p. 42-47, 2019.

Duke, N. N. et al (2020). Institutional Factors Associated with Burnout Among Assistant Professors. Teaching and Learning in Medicine, v. 32, n. 1, p. 61-70, 2020 .

Gomes, I. S., \& Caminha, I. D. O. (2014). Guide to systematic review of studies: An option for the methodology of human movement sciences. Movimento, 20(1), 395-411. https://doi.org/10.22456/1982-8918.41542

Gonçalves, T. B. et al (2011). Prevalência de síndrome de Burnout em professores médicos de uma universidade pública em Belém do Pará. Revista Brasileira de Medicina do Trabalho, v. 9, n. 2, p. 85-89, 2011.

Junior, A. G. B. et al (2017). Relação entre a síndrome de burnout e a prática docente médica. Unimontes científica, 2017.

Koche, J. C. (2011). Fundamentos de metodologia científica. 
Research, Society and Development, v. 10, n. 6, e12710615578, 2021

(CC BY 4.0) | ISSN 2525-3409 | DOI: http://dx.doi.org/10.33448/rsd-v10i6.15578

Lima, L. DE et al (2019). Prevalência de síndrome de Burnout em professores médicos de instituição de ensino superior da Paraíba. v. 19 , p. 299 -311, 2019.

Ludke, M. \& Andre, M. E. D. A. (2013). Pesquisas em educação: uma abordagem qualitativa. São Paulo: E.P.U.

Mendonça, V. L. G. DE et al (2012). Síndrome de Burnout em médicos docentes de uma instituição pública. Psicologia em Pesquisa, v. 6, n. 2, p. 90-100, 2012.

Nazari, H. et al (2016). The Prevalence of Job Stress and its Relationship with Burnout Syndrome among the Academic Members of Lorestan University of Medical Sciences. Journal of Caring Sciences, v. 5, n. 1, p. 75-84, 2016.

Pereira A. S. et al. (2018). Metodologia da pesquisa científica. [free e-book]. Santa Maria/RS. Ed. UAB/NTE/UFSM.

Pereira, R. C. et al (2019). Síndrome de Burnout em professores de medicina: revisão sistemática. EDaPECI, v. 18, n. 3, p. 115-126, 2019.

Popa-Velea, O. et al (2019). Factors associated with burnout in medical academia: An exploratory analysis of Romanian and Moldavian physicians. International Journal of Environmental Research and Public Health, v. 16, n. 13, p. 10-14, 2019.

Portilho, J. P. et al (2011). Posibles manifestaciones de síndrome de Burnout en docentes catedráticos e ocasionales de los programas de medicina y trabajo social de una universidad del eje cafetero - Colombia. n. 22, p. 1-7, 2011.

Santos, N. P. et al (2016). Docência universitária e o estresse: estressores nos cursos de enfermagem e medicina. Revista de Enfermagem da UFSM, v. 6, n. 1, p. 61,2016

Silva, L. C. N. DA et al (2020). Sindrome de Burnout entre Docentes de um Centro Universitário do Acre. ID on line REVISTA DE PSICOLOGIA, v. 14, n. 50, p. 1271-1281, 2020

Tijdink, J. K. et al (2014). Emotional exhaustion and burnout among medical professors; A nationwide survey. BMC Medical Education, v. 14, n. 1, p. 1-7, 2014.

Yao, S. M. et al (2015). Job-Related Burnout and the Relationship to Quality of Life among Chinese Medical College Staff. Archives of Environmental and Occupational Health, v. 70, n. 1, p. 27-34, 2015.

Yin, R.K. (2015). O estudo de caso. Porto Alegre: Bookman 